Psychiatre

ste, membre

de la Société

Psychanalytique de Paris. Médecin

directeur du centre de soin

psychothérapique de la Velotte de 1996 à 2012.7 chemin des Chevanney, 25000 Besançon, France. pascaledesaintemarie@ gmail.com

\title{
Du conflit d'autonomie au conflit des origines
}

\author{
Pascale de SAINTE MARIE
}

\section{Résumé}

À partir de la conflictualité inhérente à l'autonomie psychique, l'auteur explore le conflit des origines tel que le décrit Paul Claude Racamier dans l'Antœdipe, en insistant sur le rôle organisateur du deuil originaire. Elle illustre son propos par une vignette clinique et donne des pistes pour le soin.

Mots clés : autonomie, narcissisme, croissance, origines, deuil.

\section{From the conflict of autonomy to the conflict of origins}

Abstract

From the conflictuality inherent in psychic autonomy, the author explores the conflict of origins as described by Racamier in Antoedipe, emphasizing the organizing role of the original mourning. She illustrates her point with a clinical vignette and gives some tips for the treatment.

Key words: autonomy, narcissism, growth, origins, mourning.

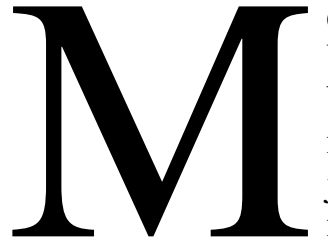

erci au professeur Pierre Vandel et à Vincent Rebière qui viennent de nous introduire dans ces journées et qui continuent aujourd'hui à faire vivre la Velotte, 50 ans déjà... Au fil des ans la Velotte a évolué, a gagné en maturité et en stabilité tout en restant fidèle à l'esprit des origines insufflé par P.-C. Racamier, esprit de coopération, de créativité partagée et d'innovation.

P.-C. Racamier a construit la Velotte pas à pas, il en a organisé le cadre et les méthodes de soin au fil de ses découvertes. $\mathrm{Il}$ a réussi à en faire un ensemble cohérent, offrant un cadre complexe et pourtant simple dans ses repères, enveloppant et ouvert sur l'extérieur, sécurisant et responsabilisant. Ce cadre prend chair et vie grâce à son fort investissement par l'équipe tout entière, c'est-à-dire l'équipe médico-soignante mais aussi les personnes s'occupant de l'animation, du secrétariat, du ménage et de la cuisine. Les fonctions sont bien

Travail soutenu par le fond de recherche scientifique de l'association ENFASA. différenciées mais sans clivage entre elles, ce qui permet une qualité d'attention et d'investissement jusque dans les détails de la vie quotidienne et la recherche soutenue d'une bonne qualité de vie ensemble. La richesse des échanges, la libre circulation de la parole, dans une équipe à petit effectif permettent une grande souplesse de fonctionnement et un vrai plaisir à travailler et à penser ensemble.

Ce cadre solide, souple et vivant, pré-investi par l'équipe comme une sorte d'appareil à penser, à penser les temps, les espaces, les comportements, les agirs, les paradoxes, les dilemmes, les contradictions, les conflits... Ce cadre offre la possibilité au patient de s'y installer, de s'y loger à sa façon à lui, singulière, et d'y inventer sa propre trajectoire.

Dans le même temps, les parents sont associés à la cure par des contacts réguliers. La coopération demandée aux parents est une des marques d'origine de la Velotte. Cela peut paraître surprenant pour un centre de soins s'adressant à des patients adultes et dont l'objectif premier est de leur permettre d'accéder à une meilleure autonomie. Mais l'autonomie est une notion plus complexe qu'il n'y paraît. 
Rembrandt, «Le retour de l'enfant prodigue » (ㄷ The State Hermitage Museum St. Petersburg, Russia).

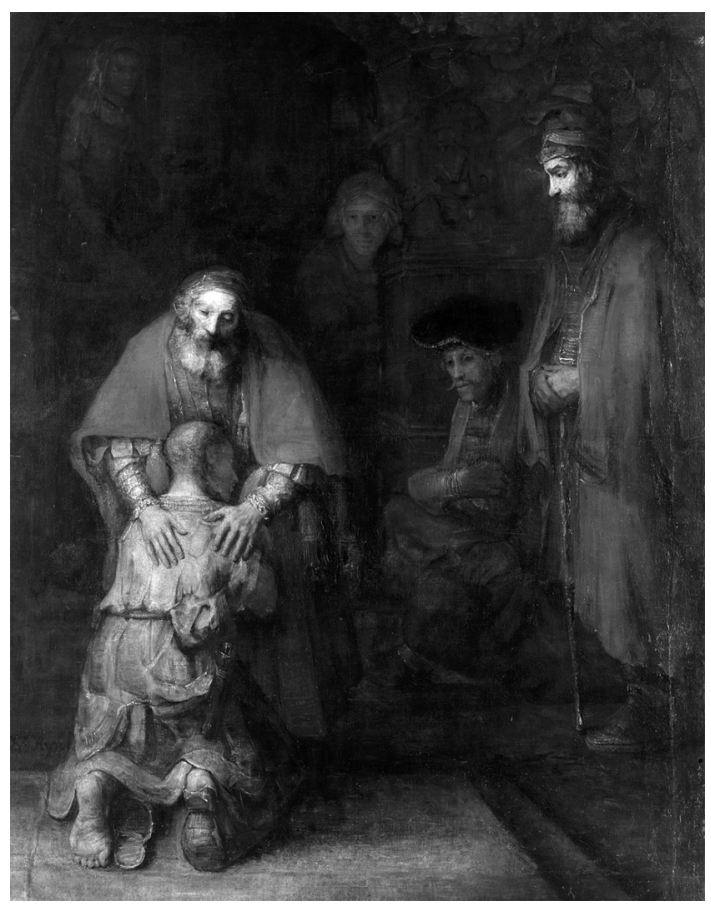

Nous n'allons pas nous attarder sur les difficultés d'autonomie pragmatiques de ces patients, qui souvent pèsent sur l'entourage et que nous connaissons bien. Elles sont intimement liées à un manque d'autonomie psychique, c'est ce dont nous allons parler maintenant. Pourquoi parler de conflit au sujet de l'autonomie?

C'est un conflit qui se joue sur l'existence même. Le fameux «être ou ne pas être». Schématiquement, je peux le formuler ainsi : nous n'avons pas demandé à naître, la vie, nous l'avons reçue de nos parents, nous n'y sommes pour rien, notre vie ne nous appartient pas. Par ailleurs, les forces de croissance qui nous habitent nous ont rendus capables de nous approprier notre existence. Notre vie, c'est à nous de l'inventer, elle nous appartient. Alors ? Notre vie nous appartient ou ne nous appartient pas ? Interrogation cruciale à l'adolescence en arrière-fond du remue-ménage provoqué par la poussée pubertaire.

P.-C. Racamier, avec l'antœdipe, mis en rapport de complémentarité avec l'œdipe, nous donne une réponse claire et positivement ambiguë. Il nous fait redécouvrir de façon nouvelle que la vie nous appartient et ne nous appartient pas, c'est-à-dire que «nous sommes depuis toujours et sans le savoir les co-auteurs de la vie qui nous est donnée... Il ne suffit pas de naître, encore faut-il construire sa naissance au monde. Il ne suffit pas d'avoir reçu la vie, encore faut-il se la donner... Ainsi va la psyché: elle reçoit ce qu'elle invente, elle crée ce qu'elle trouve » (Racamier, 1989). La résolution de ce conflit portant sur l'existence même ne va pas de soi. Nous connaissons bien l'ambivalence, qui tricote ensemble l'amour et la haine. Nous connaissons moins l'ambiguïté qui porte sur l'existence même. «Être et ne pas être » est la définition la plus condensée de l'ambiguitté. En elle se conjuguent les forces de survivance et d'anéantissement. L'emploi du terme ambiguïté peut prêter à confusion. Nous le connaissons plutôt dans son sens équivoque et douteux. Racamier l'utilise dans son acception positive comme étant la capacité de conjuguer deux éléments de nature différente et qui ne s'excluent pas mutuellement. Nous sommes les co-auteurs de la vie qui nous est donnée est une formulation ambiguë. Je crois que ce que j'ai le plus apprécié dans mon travail avec P.-C. Racamier, c'est l'énergie et l'intelligence qu'il déployait pour détruire certains schémas sclérosants qui figent la pensée : je pense au dualisme du corps et de l'esprit et au dualisme de l'inné et de l'acquis. Il me semble que ces dualismes imprègnent encore certaines de nos pratiques médicales. J'observais avec beaucoup d'intérêt et d'attention son étonnante inventivité pour sans cesse relier le psychique et le pragmatique, notamment à travers ses trouvailles comme les actions parlantes.

Revenons-en à l'autonomie. Comment ne pas rester aliéné aux parents, sans les rejeter, sans les détruire ? Le patient psychotique ne sait pas faire, il reste emmêlé dans les relations familiales et ses parents avec lui. Certains peuvent penser qu'il n'y peut pas grand-chose... la schizophrénie, c'est génétique... Nous savons bien que ce n'est pas si simple, et que le patient peut avoir sa part. Il est temps de remonter à la source, à la rencontre de l'antœdipe. Pour Racamier, l'œdipe et l'antœdipe sont les deux grandes constellations conflictuelles essentielles du fonctionnement psychique, distinctes mais en interaction permanente tout au long de la vie. Le conflit antœdipien, c'est celui des origines. Il oppose les forces visant à l' unisson narcissique avec la mère primaire et les forces visant au contraire à la séparation puis à l'autonomie. Ce sont les forces de la séduction narcissique qui s'opposent à celles de la croissance : telle est la nature du conflit. Cette configuration est complétée chez 
la mère par des aspirations similaires plus ou moins convergentes. À la différence de l'œdipe qui se déroule en intrapsychique, le conflit antœdipien des origines ne peut se dérouler et encore moins se résoudre sans la participation active de la mère. Il s'organise non pas en intrapsychique, mais en interpsychique, ce qui rend sa compréhension plus complexe. Si la mère n'est pas présente avec son bébé ou trop en difficulté face à un bébé absent du contact, l'antodipe ne pourra pas nâ̂tre, si à l'inverse elle est trop présente et exerce une emprise trop forte, l'antœdipe ne pourra se résoudre. C'est la séduction narcissique, force puissante d'aimantation mutuelle qui, dès la naissance, va permettre la rencontre entre le bébé et sa mère. Elle passe par le regard et par la peau. Racamier nous donne l'image du bébé qui tète le sein et distingue ce qui se passe au niveau de la bouche suceuse du bébé et ce qui se passe au niveau de son regard plongé dans celui de sa mère qui le regarde et le porte. La pulsion passe par la bouche, mais l'investissement antœdipien passe par le regard. «Lorsque la bouche $d u$ bébé travaille au sein, son eil travaille au monde. » (Racamier, 1989). Le bébé se nourrit au sein et en même temps au sein des échanges. L'investissement antœdipien passe aussi par la peau : elle enveloppe et elle sépare; sa fonction est différenciatrice. Cela laisse présager de l'importance de l'antœdipe dans la construction des enveloppes psychiques et dans l'organisation des limites. La séduction narcissique, ce bain d'admiration mutuelle, cette aimantation puissante peut donc présenter deux versions différentes et distinctes. D'abord voisines elles se séparent, s'écartent et vont bientôt s'opposer : la séduction narcissique peut être ouverte ou fermée. Dans les bons cas, quand la séduction est ouverte, l'enfant poussé par la croissance, se déprend progressivement de l'unisson narcissique avec sa mère et se détourne de celle-ci, il lui tourne le dos. Alors la mère, plutôt que de maintenir à tout prix une relation fusionnelle, déplace son investissement vers les progrès de l'enfant et accompagne son mouvement vers l'autonomie. Ouverte, la séduction narcissique peut prendre fin. Elle a été nécessaire, indispensable et plus elle aura été ajustée, plus elle pourra aisément se terminer. Elle va finir par se résoudre en se fondant au sein du moi dont elle va nourrir l'identité. Par contre, si elle est fermée, elle prend la forme d'une emprise. Cette emprise peut être entretenue, par exemple, par une mère « trop bonne » qui anticipe tous les besoins de l'enfant pour qu'il n'ait ni à réclamer ni à souffrir, ou qui isole son bébé de toute présence étrangère (dont le père) pour que rien ne vienne troubler leur relation exclusive. Cette relation d'emprise narcissique peut aller jusqu'à s'opposer fortement à la croissance psychique de l'enfant et à son besoin d'autonomie. Elle devient alors interminable, créant une faille dans le narcissisme de l'enfant qui risque de se trouver en difficulté face aux séparations et aux exigences d'autonomie rencontrées au cours de la vie. Il y a une bifurcation dans l'antœdipe, avec deux voies possibles qui peuvent aller vers le meilleur, ou vers le pire... vers l'autonomie ou vers l'aliénation. Le chemin vers l'autonomie ne va pas de soi, c'est un chemin à trouver et à ouvrir, il y a une traversée à faire et surtout, cette traversée ne peut se faire sans la participation active de la mère, sans son accompagnement.

L'enfant quand il se détourne de sa mère, il la perd, il accepte de la perdre. Il aura à en faire un deuil, le deuil d'une illusion de toutepuissance et de toute appartenance. «Il s'est détourné d'une mère qui est comme une atmosphère: il la déplore; il découvre une mère qui est un objet: il la désire. Tel est le deuil originaire: condition de la découverte de l'objet en tant que tel. Du même coup le monde se divise en deux parts l'interne et l'externe. Ces parts ne sont pas clivées, elles restent reliées, mais distinctes. » (Racamier, 1992).

Encore faut-il que la mère l'accepte et qu'elle accompagne le mouvement. C'est le déclenchement du processus de deuil originaire qui permet la traversée. Il est l'organisateur interne de l'antœdipe et son seuil est essentiel. C'est par lui que s'instaure la différence entre l'autre et soi, entre hier et demain, c'est une découverte payée du prix d'une perte. Le deuil originaire est un processus qui une fois enclenché, se poursuit tout au long de la vie. Il s'oppose à la confusion des êtres et des générations. Racamier fait du deuil originaire l'organisateur central du conflit des origines. « Le deuil originaire désigne le processus psychique fondamental par lequel le moi, dès ses prémices, avant même son émergence et jusqu'à la mort, renonce à la possession totale de l'objet, fait son deuil d'un unisson narcissique absolu et d'une constance de l'être indéfinie, et par ce deuil même, qui fonde ses origines, opère la découverte ou l'invention 
de l'objet, et par conséquent de soi, grâce à l'intériorisation. Le moi établit donc ses origines en reconnaissant qu'il n'est pas le mấtre absolu de ses origines. Il se découvre en se perdant: tel est le paradoxe identitaire. Pour être encore plus concis, nous pourrions dire que le deuil originaire constitue la trace ardue, vivante et durable, de ce que l'on accepte de perdre comme prix de toute découverte. » Ce travail ne se fait pas d'un coup, il se fait progressivement, mais Racamier insiste : "Rien après le deuil originaire ne sera plus jamais comme avant... Si ce travail n'est pas entamé, jamais l'objet ne sera vraiment repéré, investi et désiré en tant que tel. » (Racamier, 1992).

Et nous allons voir que c'est, entre autres, sur ce travail du deuil originaire, passage obligé sur le chemin de l'autonomie, que butent les patients psychotiques.

Je vais maintenant vous présenter une brève vignette clinique qui montre l'apparition du travail de deuil originaire chez une patiente souffrant de schizophrénie. Il s'agit de Rachel. Rachel est en soin depuis un peu plus de 2 ans à la Velotte, lorsque survient l'événement dont je vais vous parler. Auparavant, quelques mots sur son admission. Avant de venir nous rencontrer pour la première fois avec ses parents, elle avait été hospitalisée quelques mois en hôpital psychiatrique, après avoir été récupérée de justesse alors qu'elle était en train de se défenestrer de son studio situé au $8^{\mathrm{e} e ́ t a g e}$ d'une tour. Elle avait alors dit qu'elle fuyait un fantôme qui la poursuivait. Lors de la préadmission, reçue seule dans un premier temps, Rachel était très discordante, envoyant des messages paradoxaux qui rendaient le contact avec elle particulièrement étrange : elle pouvait par exemple vous décocher un regard furieux et agressif tout en vous faisant un doux sourire. Elle semblait sans cesse vaciller comme au bord d'un précipice. Elle n'avait supporté que quelques minutes d'entretien, le temps de m'injecter une angoisse massive, et de me dire qu'elle voulait... ne pas venir. Puis elle était sortie précipitamment du bureau. J'avais ensuite reçu les parents seuls.

Ses parents, divorcés depuis longtemps, étaient venus accompagner leur fille ensemble et se présentaient lors de l'entretien, comme un couple parfaitement uni, heureux de se retrouver et d'accord sur le fait que leur fille avait besoin de se soigner. La mère m'expliqua qu'elle était devenue une sportive de haut niveau lorsqu'elle avait rencontré M. P. (le père de Rachel). Elle était rapidement tombée enceinte. Elle fut alors poussée par son entourage à avorter si elle voulait continuer à participer aux championnats prévus. Le père n'avait pas pris position, disant simplement qu'il accepterait son choix. Elle avait décidé d'avorter mais sans arriver à mettre sa décision à exécution. Ils avaient donc " laissé naître Rachel ». C'est ainsi qu'elle l'avait formulé. Et ça avait été l'enfer, Rachel pleurait beaucoup. Ils ne savaient pas s'occuper d'un bébé et ç'avait été la fin de sa carrière sportive. Lorsque je les ai reçus ensuite avec Rachel, la belle entente des parents avait disparu, ils étaient en désaccord permanent au sujet de leur fille, sauf sur le fait qu'elle devait venir à la Velotte le plus rapidement possible. Rachel, elle, ne voulait pas. Elle se montrait ragaillardie entre ses deux parents et plus consistante. J'observais qu'elle remettait de l'huile sur le feu dès que la tension semblait baisser entre eux. Elle avait alors une lueur intense dans le regard, cette lueur si particulière que l'on observe chez les patients psychotiques en pleine jouissance de toute-puissance et d'auto-engendrement. Finalement, après quelques mois et bien des complications, des allers et retours et une première entrée avortée, Rachel s'était décidée à entrer à la Velotte et à s'y intégrer tant bien que mal ; mais la séparation et l'éloignement d'avec les parents restaient très difficiles. Lors des visites elle se « recollait » immédiatement à eux. Un peu plus de deux ans ont passé quand nous arrivons à la période qui nous intéresse. Il est toujours très difficile d'entrer en relation de façon authentique avec Rachel. Soit elle est très adhésive, se collant à l'avis du dernier qui a parlé, soit elle se montre agressive et rejetante, persécutée par l'entourage. Pourtant nous pensons qu'elle progresse. Déjà par le fait qu'elle a investi un carré de jardin dont elle s'occupe avec beaucoup de soin. Elle lui a fait une bordure. Elle y cultive des salades, des tomates cerises et des fleurs. Elle le protège contre les envahisseurs. Elle distribue ses tomates aux uns et aux autres... Cela nous fait penser à une ébauche d'espace personnel encore extérieur mais qui lui appartient. C'est un espace que nous respectons et dont nous pouvons parler avec elle. Par ailleurs, elle a accepté une série d'enveloppements humides qu'elle apprécie et pendant lesquels il est devenu possible de parler avec elle. Les soignants ont pu notamment aborder la question 
des vêtements qu'elle s'acharne à porter et qui ne sont pas à sa taille, soit trop petits, soit trop grands. Elle leur apprend que ce sont des vêtements ayant appartenu à son père, ou à sa mère, ou à un ami de ses parents qui est décédé. Elle commence à penser qu'elle pourrait s'en défaire et s'acheter des vêtements à elle. Cela nous laisse penser qu'elle progresse, mais paradoxalement dans le même temps elle devient de plus en plus persécutée et violente. Nous sentons l'amorce d'une crise.

C'est dans ce contexte qu'arrive ce moment particulier dont je voulais vous parler. Nous sommes réunis pour la réunion de groupe hebdomadaire du vendredi matin (tous les patients et l'équipe médico-soignante presque au complet).

Les patients peuvent s'inscrire pour prendre la parole, ce que fait Rachel ce jour-là. Quand c'est son tour de parler, elle reste silencieuse, visiblement émue, ce qui est très inhabituel... Je lui demande si elle peut nous dire ce qui la remue ainsi. Elle nous dit alors qu'elle se voit comme une momie à laquelle on enlève ses bandelettes les unes après les autres et qu'audessous... il n'y a qu'un trou noir... et elle se met à pleurer.

C'est la première fois qu'elle nous apparaît authentique. Nous sommes tous très bouleversés. Nous qualifions le fait qu'elle puisse nous parler de ce qu'elle ressent de façon si personnelle et authentique, que ce trou noir peut lui faire peur, mais c'est aussi un espace inconnu qui s'ouvre qui peut lui permettre de se trouver et de naître à elle-même. Avec la perte de ses bandelettes, elle perd un peu de ses parents, c'est triste, elle peut se sentir seule mais nous sommes là pour la soutenir et l'aider dans ce passage difficile. C'est à partir de ce moment que nous avons eu le sentiment que Rachel commençait à entrer vraiment dans sa cure, dans sa peau et dans sa tête. C'était un début, il y avait un avant et un après, c'est-à-dire l'entrée aussi dans une temporalité.

Les deux premières années de sa cure lui ont sûrement permis de se recharger narcissiquement au sein des échanges à la Velotte et d'effectuer un travail plus ou moins souterrain d'intégration des limites et du cadre de soin. Les enveloppements humides lui ont permis de quitter ses vêtements d'emprunt pour se laisser envelopper par un soignant et une soignante dans des draps glacés, paradoxalement protecteurs. En plus, cela lui a permis d'expérimenter que c'était sa chaleur à elle, venant de l'intérieur d'elle-même, qui réchauffait les draps glacés. La vie en elle...

Son aspiration vers un peu d'autonomie s'est réveillée et est entrée alors en contradiction avec le collage/mélange avec les parents. Le port des vêtements formait un verrou, le fait qu'il commence à sauter rend la paradoxalité moins serrée, plus visible dans la cure. Elle nous la fait vivre: en effet, nous trouvons qu'elle progresse, et dans le même temps elle se montre de plus en plus persécutée, agressive et invivable dans le groupe. Elle veut quitter la Velotte mais ne supporte pas qu' on en parle et s'incruste. C'est bien d'autonomie qu'il s'agit mais comment trouver la bonne porte ? La paradoxalité s'ouvre et prend la forme d'un dilemme qui apparaît souvent lorsqu'un patient a à faire un pas vers l'autonomie qu'il redoute. Il veut rompre sa cure tout en refusant de partir. Nous sommes pris en tenaille. La solution ne pourra venir que du patient, à nous de lui exposer le dilemme et de l'aider à trouver une solution. Il aura toujours quelques marches à descendre, quelque perte à accepter. Perdre de sa mégalomanie et de sa toute-puissance n'est pas chose facile. Rappelons-le : c'est un travail de deuil qui mène à l'autonomie. Et la perte précède la découverte. On sait ce que l'on perd, mais on ne sait pas ce que l'on va y gagner...

J'aurais beaucoup d'histoires à raconter sur ces moments d'ouverture au travail du deuil originaire. Ils sont présents dans toutes les cures des patients psychotiques, quand ils sont vraiment engagés dans un processus de soin. Ils sont plus ou moins spectaculaires parfois très discrets, toujours émouvants de par la tristesse qui peut enfin être partagée.

Revenons-en à Rachel.

Rachel s'est organisée en se ligaturant de façon très paradoxale et serrée avec ses parents ; nous l'avons observé dans les entretiens préliminaires et avec le port des vêtements, et cela au prix de son inexistence. Ces vêtements étaient des sortes d'objets fétiches, venant verrouiller un clivage sûrement très profond chez elle, clivage qui la prive d'une partie d'ellemême, qui vient recouvrir un déni portant sur son existence même, ce clivage déborde son moi en mal de limites, cette faille se prolonge avec les parents les protégeant de leurs propres dénis. Les parents participent au verrouillage en la laissant s'habiller avec leurs vêtements et sans rien en dire.

Cet épisode de la momie marqua donc un moment important de sa cure, un seuil franchi. Mais une crise bien plus redoutable se profila 
peu de temps après. C'est Rachel qui l'amorça et elle secoua toute sa famille. C'était encore une histoire d'enveloppe, mais plus précisément d'anti-enveloppe et d'espace antipersonnel. Cette crise s'amorça quand Rachel se mit à parler de son studio.

Cela fait déjà trois ans que Rachel est à la Velotte et le studio est toujours loué, bien que Rachel n'y soit jamais retournée. Les parents y tiennent, disant que c'est «son espace à elle », mais Rachel dit qu'elle veut s'en débarrasser et qu'elle ne veut plus y aller.

Lors de la pré-admission nous avions découvert qu'il avait une fonction bien particulière. Il est loué et payé par le grand-père paternel avec lequel Monsieur P. n'a plus aucun contact mais avec lequel, par contre, Madame M. est en bon terme. C'est Rachel qui reçoit les allocations ; ce qui lui fait un peu d'argent de poche. La grand-mère maternelle de Rachel habite dans l'immeuble voisin et Madame M. a demandé au facteur de déposer le courrier de Rachel dans la boîte aux lettres de sa propre mère, ce qui lui permet de surveiller le passage des courriers administratifs que Rachel pourrait négliger. Le studio a été meublé par Monsieur $P$. avec l'aide financière de sa mère (la grandmère paternelle). Depuis peu, il a coupé les ponts avec elle ; il la décrit comme tyrannique et cherchant à exercer une emprise sur Rachel. Ce studio semble donc avoir un rôle très particulier. Sorte de crypte, d'espace non-espace, d'objet paradoxal structuré à partir des dénis familiaux. Sous prétexte d'offrir un espace à Rachel, il amalgame la mère, le grand-père paternel, la grand-mère maternelle, le père et la grand-mère paternelle dans une sorte de forteresse narcissique, de trou noir, machine à absorber les conflits et les menaces d'endeuillement et de séparation. Cela évoque les équivalents d'inceste décrits par Racamier. Rachel est au centre des manipulations familiales inconscientes, à travers les manipulations d'argent, de courrier, de mise en situation dangereuse. «La certitude qui déjà s'est imposée à nous est que l'équivalent d'inceste, quelle que soit son apparence, exerce la fonction d'un objet fétiche. Mais ce n'est pas un fétiche individuel; c'est un fétiche partagé. Cet objet si banal en apparence, et si intimement déconnecté du réseau représentationnel, cet objet est l'instrument d'un lien entre ceux qui le détiennent. Non pas vraiment d'un lien mais d'une ligature. C'est bien là ce qui lui vaut auprès de ses détenteurs, un si tenace attachement. »(Racamier, 1995).
Nous avions été convaincus, dès le début, qu'il ne fallait pas toucher de nous-mêmes à ce studio, sachant, par expérience, que s'approcher d'un tel lieu était comme s'aventurer en terrain miné où tout peut exploser. C'est pourquoi, nous n'en avions pas reparlé, préférant que le sujet soit abordé en temps voulu. Maintenant Rachel voulait s'en défaire. Elle écrivit à son grand-père pour lui dire qu'il pouvait arrêter la location du studio car elle n'irait plus jamais vivre dedans. Il s'ensuivit des disputes très violentes au sein de la famille et avec Rachel, pour des questions d'argent, de meubles, de déménagement, de ménage... Rachel devenait incontrôlable et suicidaire et les parents accusateurs.

Je passe toutes les étapes de cette crise qui dura plusieurs mois et faillit à plusieurs reprises provoquer la rupture de la cure. Finalement Rachel accepta de se faire hospitaliser. Pendant ce temps nous poursuivions les contacts téléphoniques hebdomadaires avec chacun des parents et nous les avons reçus plusieurs fois séparément. Ils ont pu parler de leur histoire, des conflits familiaux, aborder des souffrances anciennes dont ils n'avaient jamais pu parler, des deuils, jusque-là gelés, ont pu commencer à s'ouvrir. Petit à petit le climat s'est apaisé. Au bout de quelques mois, Rachel est revenue à la Velotte dans de bien meilleures conditions.

\section{Conclusion : quelques réflexions sur l'accompagnement thérapeutique}

\section{Tout d'abord : l'importance du cadre}

Que ce soit en cabinet ou en institution, je pense qu'il est essentiel que le cadre soit clair, pré-investi par les médecins et les soignants, qu'il soit explicité et repérable avec des règles compréhensibles et valables pour tout le monde. Il est important que l'entrée dans le cadre soit marquée par une transaction, le patient aura à y entrer pour pouvoir en sortir. Nous lui laisserons ensuite le temps de l'expérimenter, de l'éprouver et d'apprendre à s'en servir.

Je pense aussi à l'importance des espaces transitionnels, des espaces de jeu qui permettent aux patients psychotiques de découvrir ou de redécouvrir un espace perdu. Leurs dénis portent sur la réalité extérieure et sur la réalité intérieure, les deux vont toujours ensemble. L'espace intermédiaire, ambigu selon Raca- 
mier, est quasi inexistant. Ces trois espaces de réalité sont amputés au profit d'un quatrième espace, un « espace à délire », plus ou moins habité par le délire, mais toujours empli de jouissance narcissique mégalomaniaque. Les fantasmes, non-fantasmes, d'auto-engendrement et de dés-engendrement y sont à l'œuvre. Tout ce qui sera ouvert dans un espace transitionnel, ambigu, sera gagné sur l'espace, non-espace du délire. D'où l'intérêt des jeux, des sorties, des activités partagées et dans le cadre du soin proprement dit, des actions parlantes qui sont proprement ambiguës.

- Il y a un autre double déni, important à connaître. C'est celui des capacités et des difficultés du patient. Ce double déni, le patient le cultive, il se vit tout-puissant et maître du monde. Quant à ses difficultés il pense qu'elles ne sont pas en lui mais qu'elles viennent du dehors. Ce double déni, nous pouvons y répondre par une double qualification et de ses capacités et de ses difficultés.

En après coup je repense au jardinet que Rachel avait su se construire au sein de la Velotte. Elle s'était construit là un espace transitionnel qui était et à elle et à la Velotte, ce qui l'a sûrement beaucoup aidée à retrouver de l'air et des forces. Rachel nous a montré ses capacités avec son jardin et sa souffrance profonde avec l'image de la momie. Il était important de les qualifier toutes les deux.

- Un mot enfin pour terminer sur notre capacité à faire des deuils.

Nous avons vu que le patient attend de nous une guérison magique. Et les psychoses ont ce mérite qui est de nous montrer à la loupe ce qui passe habituellement inaperçu. Avec elles, il est impossible de passer à côté du problème que nous pose la passion de vouloir guérir. Les psychoses nous mettent face à un écartèlement, un dilemme : la nécessité de guérir à tout prix et l'inanité de vouloir guérir. Les patients, et encore plus leurs parents, souvent nous y poussent. Ils attendent une guérison qui leur permettrait de retrouver l'enfant idéal auquel ils ne peuvent renoncer. Les patients, eux, ont une peur terrible du changement. Le changement, nous l'avons vu, consiste avant tout en une perte, une perte vécue avant la découverte. Alors... « Plutôt mourir que de perdre la vie » (Racamier, 1992).
Changer, c'est perdre une armature défensive qui aura permis de survivre. Je me souviens de la belle formulation paradoxale d'une de nos patientes, qui nous avait dit dans un moment de vertige face au changement: «J'ai peur de perdre ce que je ne suis pas ! » Il est donc important de renoncer à guérir, de laisser le patient prendre l'initiative des moments mutatifs. C'est l'équivalent de l'anticipation maternelle qui nous permet d'accompagner les crises et de leur donner sens. Entre-temps, nous valoriserons les petits pas, qui seuls permettent d'avancer réellement vers l'autonomie. Et si une «guérison» finit par se produire, elle sera donnée par surcroît.

- Un petit mot pour conclure sur le tableau de Rembrandt (voir page 305), « Le retour de l'enfant prodigue », qui figure sur le programme.

Rembrandt a une soixantaine d'années quand il peint cette œuvre. C'est un homme usé par les faillites et les deuils. Il pleure encore son fils Titus et il va mettre toute son intériorité à peindre des retrouvailles. C'est un père tendre qui accueille son fils qu'il avait perdu. L'une de ses mains semble féminine et l'autre masculine, et l'on observe l'attitude maternelle du père dans sa façon d'accueillir sur son ventre son fils qui vient s'y nicher. Le fils est peint comme une sorte de condamné avec sa tête rasée, son pied nu laisse deviner qu'il est devenu pauvre, et qu'il a fallu qu'il perde sûrement beaucoup de sa superbe pour revenir ainsi chez son père, débarrassé de ses fausses richesses.

Je trouve que c'est une belle image du travail de deuil et de l'ambiguïté retrouvée.

\section{LIENS D'INTÉR̂̂T}

L'auteur déclare n'avoir aucun lien d'intérêt concernant les données publiées dans cet article.

\section{RÉFÉRENCES BIBLIOGRAPHIQUES}

Racamier P.-C. (1989). Antcedipe et ses destins. Paris : Apsygée Editions.

Racamier P.-C. (1992). Le génie des origines. Psychanalyse et psychose. Paris : Éditions Payot. Racamier P.-C. (1995). L'inceste et l'incestuel. Paris: Les Éditions du Collège. 